\title{
LOSSLESS IMAGE COMPRESSION USING NEW BIORTHOGONAL WAVELETS
}

\author{
M. Santhosh ${ }^{1}$, Dr. B. Stephen Charles ${ }^{2}$ and Dr. M N Giriprasad ${ }^{3}$ \\ ${ }^{1}$ Asso. Prof., Dept. of ECE, SSCET, Kurnool, India \\ ${ }^{2} \mathrm{M}$ Principal, SSCET, Kurnool, India \\ ${ }^{3}$ HOD, Dept. of ECE, JNTUA, Anantapuramu, India
}

\begin{abstract}
Even though a large number of wavelets exist, one needs new wavelets for their specific applications. One of the basic wavelet categories is orthogonal wavelets. But it was hard to find orthogonal and symmetric wavelets. Symmetricity is required for perfect reconstruction. Hence, a need for orthogonal and symmetric arises. The solution was in the form of biorthogonal wavelets which preserves perfect reconstruction condition. Though a number of biorthogonal wavelets are proposed in the literature, in this paper four new biorthogonal wavelets are proposed which gives better compression performance. The new wavelets are compared with traditional wavelets by using the design metrics Peak Signal to Noise Ratio (PSNR) and Compression Ratio (CR). Set Partitioning in Hierarchical Trees (SPIHT) coding algorithm was utilized to incorporate compression of images.
\end{abstract}

\section{KEYWORDS}

biorthogonal wavelets, basis function, filters, compression.

\section{INTRODUCTION}

In this section the details regarding need of wavelet and wavelet formulation is presented. The windowed Fourier transform introduces a scale (the width of the window), and analyses the signal from the point of view of this scale. If the signal has important frequency details outside of the scale, one will have problems in the signal analysis:

- If the signal details are much smaller than the width of the window, one will have a problem: the details will be detected but the transform will not localize them.

- If the signal details are larger than the width of the window they will not be detected properly.

To solve this problem when one analyzes a signal using the windowed Fourier transform, they must define a transform which is independent of scale [1]. This transform should not use a fixed scale, but the scale should vary. The scale is defined by the width of the modulation function. Therefore we must use a modulation function which does not have a fixed width. Moreover the function must have good time localization [2]. To achieve this, start from a function $\Psi(\mathrm{t})$ as a candidate of a modulation function, and we obtain a family of functions from $\Psi$ by varying the scale: We fix $p \geq 0$ and for all $s \in R, s \neq 0$, we define

$$
\Psi \mathrm{s}(\mathrm{u})=|\mathrm{s}|-\mathrm{p} \Psi(\mathrm{u} / \mathrm{s})
$$


Signal \& Image Processing : An International Journal (SIPIJ) Vol.4, No.6, December 2013

If $\Psi$ has width $T$ then the width of $\Psi$ s is sT. The modulation of the function $\Psi$ by the factor $|s|-2$, increases its amplitude when the scale s decreases and vice-versa. In terms of frequencies, we can state: For small scales s, $\Psi$ s has high frequencies, and as s increases the frequency of $\Psi \mathrm{s}$ decreases [3][4]. This fact is illustrated in Figure 1.

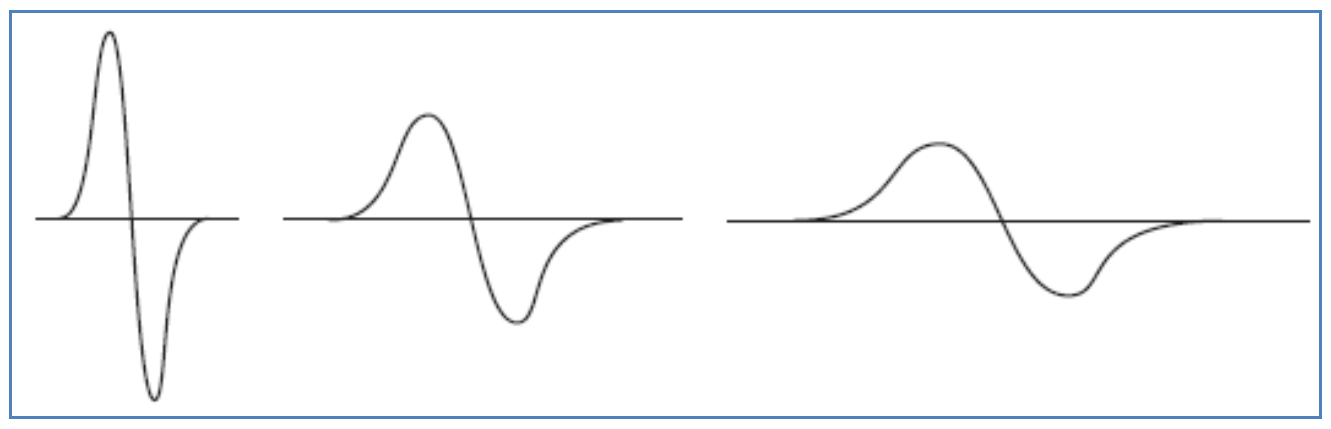

Figure 1. Scales of a function: (a) $\mathrm{s}<1$; (b) $\mathrm{s}=1$; (c) $\mathrm{s}>1$.

Analogous to what we did with the windowed Fourier transform of a function, we need to localize each function $\Psi_{s}$ in time. For this we define for each $t \in R$ the function

$$
\psi_{s, t}(u)=\psi_{s}(u-t)=|s|^{-p} \psi\left(\frac{u-t}{s}\right)=\frac{1}{|s|^{p}} \psi\left(\frac{u-t}{s}\right)
$$

Note that if $\Psi \in \mathrm{L} 2(\mathrm{R})$, then $\Psi \mathrm{s}, \mathrm{t} \in \mathrm{L} 2(\mathrm{R})$, and

$$
\left\|\psi_{s, t}\right\|^{2}=|s|^{1-2 p}\|\psi\|^{2}
$$

By taking $\mathrm{p}=1 / 2$, we have $\left\|\psi_{s, t}\right\|=\|\psi\|$

Now we can define a transform on L2(R) in a similar way that we defined the windowed Fourier transform, using functions from the family $\Psi \mathrm{s}, \mathrm{t}$ as modulating functions. More precisely, we have

$$
\tilde{f}(s, t)=\int_{-\infty}^{+\infty} f(u) \psi_{s, t}(u) d u=\left\langle\psi_{s, t}, f>\right.
$$

This transform is known by the name of the wavelet transform. The wavelet transform is in use to represent different kinds of signals including multi-dimensional signals [5][6]. Depending on specific application, a number of wavelets are proposed in the literature. In this paper four new biorthogonal wavelets are proposed which give better compression performance when compared to that of well-known traditional wavelets [7][8]. The rest of the paper is organized as follows. In the Section II, the details of biorthonality are presented. In section III, the wavelet functions and associated filters are presented. Section IV presents the simulation results of proposed wavelets on image compression. Section $\mathrm{V}$ concludes this paper.

\section{BIORTHOgONAL WAVELETS}

Biorthogonal wavelets have most of the qualities of orthogonal wavelets; with the advantage of being more flexible. There are many more biorthogonal wavelets than orthogonal ones. For these reason, they make possible a variety of design options and constitute class of wavelets most used in practical applications. Biorthogonal wavelets can have symmetry. They are associated with perfect analysis/reconstruction filter banks. Biorthogonal wavelets constitute a generalization of 
Signal \& Image Processing : An International Journal (SIPIJ) Vol.4, No.6, December 2013

orthogonal wavelets [4]. Under this framework, instead of a single orthogonal basis, a pair of dual biorthogonal basis functions is employed: One for the analysis step and the other for the synthesis step, i.e. we have reciprocal frames. The two pairs of scaling function and wavelet, $\phi, \psi$ and $\tilde{\phi}, \widetilde{\psi}$, are defined recursively by the two pairs of filters $\mathrm{m} 0, \mathrm{~m} 1$, and $\tilde{m}_{0}, \tilde{m}_{1}$. In the frequency domain these relations are

$$
\begin{aligned}
& \tilde{\phi}(w)=m_{0}(w / 2) \tilde{\phi}(w / 2), \tilde{\psi}(w)=m_{1}(w / 2) \tilde{\phi}(w / 2) \\
& \tilde{\tilde{\phi}}(w)=\hat{m}_{0}(w / 2) \tilde{\tilde{\phi}}(w / 2), \tilde{\widetilde{\psi}}(w)=\hat{m}_{1}(w / 2) \tilde{\widetilde{\phi}}(w / 2)
\end{aligned}
$$

where

$$
\begin{aligned}
& m_{0}(w)=\frac{1}{\sqrt{2}} \sum_{k} h_{k} e^{-i k w}, m_{1}(w)=\frac{1}{\sqrt{2}} \sum_{k} g_{k} e^{-i k w} \\
& \tilde{m}_{0}(w)=\frac{1}{\sqrt{2}} \sum_{k} \tilde{h}_{k} e^{-i k w}, \tilde{m}_{1}(w)=\frac{1}{\sqrt{2}} \sum_{k} \tilde{g}_{k} e^{-i k w}
\end{aligned}
$$

The biorthogonality condition in the frequency domain is equivalent to

$$
\begin{array}{ll}
\sum_{k} \tilde{\tilde{\phi}}(w+k 2 \pi) & \tilde{\phi}^{\prime}(w+k 2 \pi)=1 \\
\sum_{k} \tilde{\tilde{\psi}}(w+k 2 \pi) & \tilde{\psi}^{\prime}(w+k 2 \pi)=1 \\
\sum_{k} \tilde{\tilde{\psi}}(w+k 2 \pi) & \tilde{\phi}^{\prime}(w+k 2 \pi)=0 \\
\sum_{k} \tilde{\tilde{\phi}}(w+k 2 \pi) & \tilde{\psi}^{\prime}(w+k 2 \pi)=0
\end{array}
$$

for all $\mathrm{w} \in \mathrm{R}$. This means that the filters $\mathrm{m} 0, \mathrm{~m} 1$ and their duals have to satisfy

$$
\begin{aligned}
& \tilde{m}_{0}(w) \tilde{m}_{0}{ }^{\prime}(w)+\tilde{m}_{0}(w+\pi) \tilde{m}_{0}{ }^{\prime}(w+\pi)=1 \\
& \tilde{m}_{1}(w) \tilde{m}_{1}{ }^{\prime}(w)+\tilde{m}_{1}(w+\pi) \tilde{m}_{1}{ }^{\prime}(w+\pi)=1 \\
& \tilde{m}_{1}(w) \tilde{m}_{0}{ }^{\prime}(w)+\tilde{m}_{1}(w+\pi) \tilde{m}_{0}{ }^{\prime}(w+\pi)=0 \\
& \tilde{m}_{0}(w) \tilde{m}_{1}{ }^{\prime}(w)+\tilde{m}_{0}(w+\pi) \tilde{m}_{1}{ }^{\prime}(w+\pi)=0
\end{aligned}
$$

The set of equations above can be written in matrix form as

$$
\left(\begin{array}{cc}
\tilde{m}_{0}(w) & \tilde{m}_{0}(w+\pi) \\
\tilde{m}_{1}(w) & \tilde{m}_{1}(w+\pi)
\end{array}\right)\left(\begin{array}{cc}
m_{0}(w) & m_{1}(w) \\
m_{0}(w+\pi) & m_{1}(w+\pi)
\end{array}\right)^{\prime}=\left(\begin{array}{ll}
1 & 0 \\
0 & 1
\end{array}\right)
$$

or

$$
\tilde{M}(w)\left[M^{T}(w)\right]^{\prime}=I
$$


Signal \& Image Processing : An International Journal (SIPIJ) Vol.4, No.6, December 2013

where $\mathrm{M}$ is the modulation matrix $M=\left(\begin{array}{ll}m_{0}(w) & m_{0}(w+\pi) \\ m_{1}(w) & m_{1}(w+\pi)\end{array}\right)$ and $\mathrm{I}$ is the identity matrix.

\section{Proposed Wavelets}

The phi $(\Phi)$ and $\operatorname{psi}(\Psi)$ functions associated with the new wavelets and the four filters associated with new wavelets are plotted in the Figures 2, 3, 4 and 5. In the first wavelet the decomposition functions are somewhat similar to that of Haar. But the value during non-zero period is not constant; it is decaying from 1 to 0.9 . The reconstruction function is smooth and it is a sinusoidal variation.
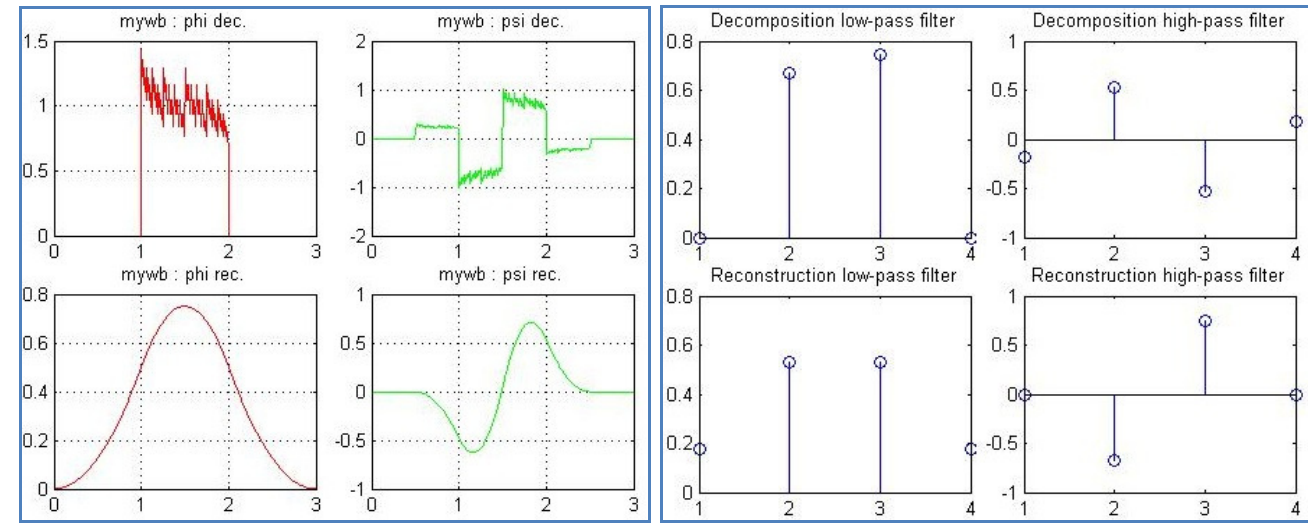

Figure 2. New Wavelets: phi, psi functions and four filters associated with NBior1
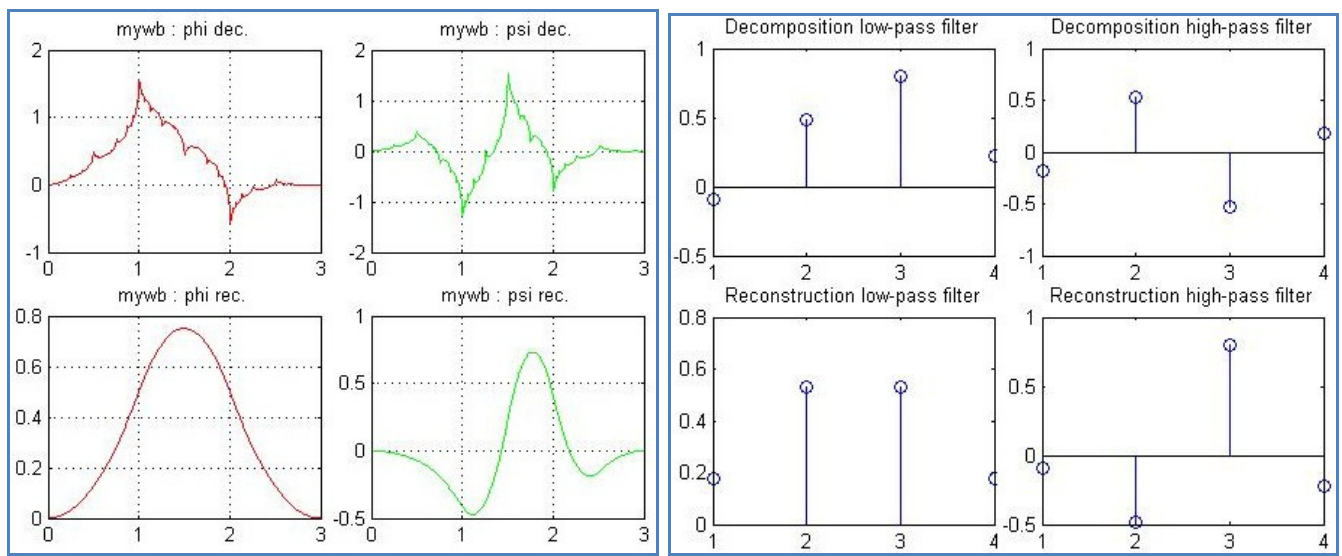

Figure 3. New Wavelets: phi, psi functions and four filters associated with NBior2

In the second wavelet, the same reconstruction stage as it is in first wavelet is used. The decomposition stage is a sharp and short wave that is supposed to capture dissimilar neighbourhood values in the input data. The decomposition stage of third wavelet is the same as that of reconstruction stage of second wavelet. In the fourth wavelet both the decomposition and reconstruction stages sharp functions are used.

\section{Simulation Results}

In this section, the performance of proposed wavelets was presented. Two categories of images are considered: multi-gray level images and binary images. In the first category both gray scale 
Signal \& Image Processing : An International Journal (SIPIJ) Vol.4, No.6, December 2013

and colour images are considered. PSNR and CR values obtained with new wavelets on multigray level images are given in the tables 1,2, 3 and 4 respectively.
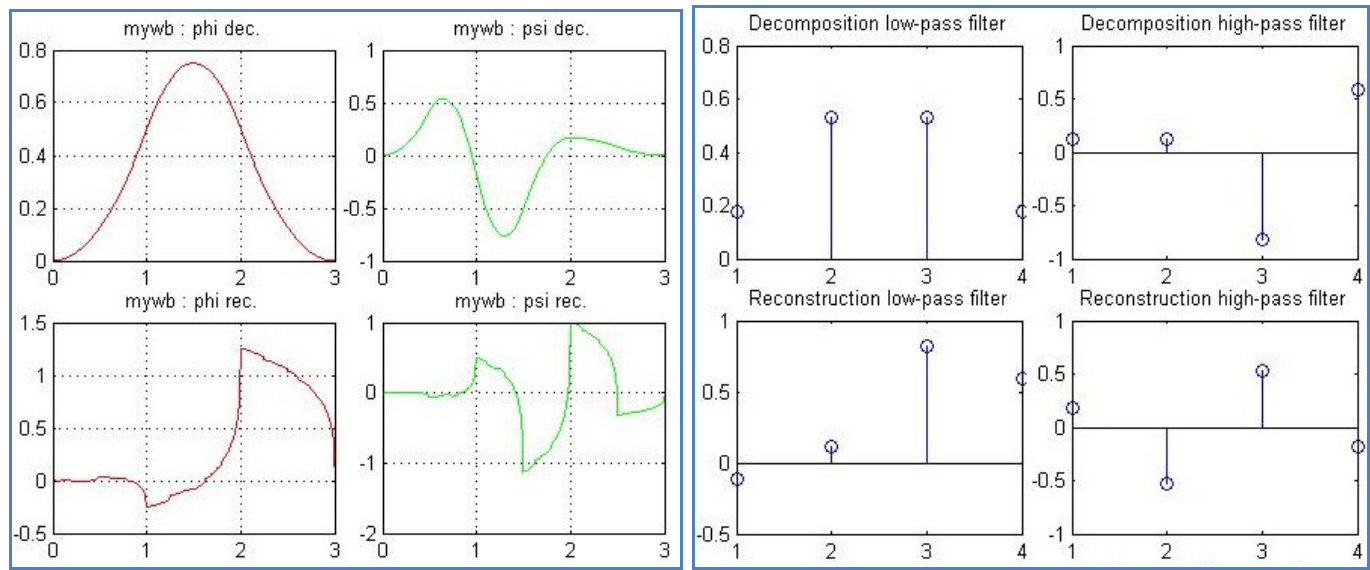

Figure 4. New Wavelets: phi, psi functions and four filters associated with NBior3
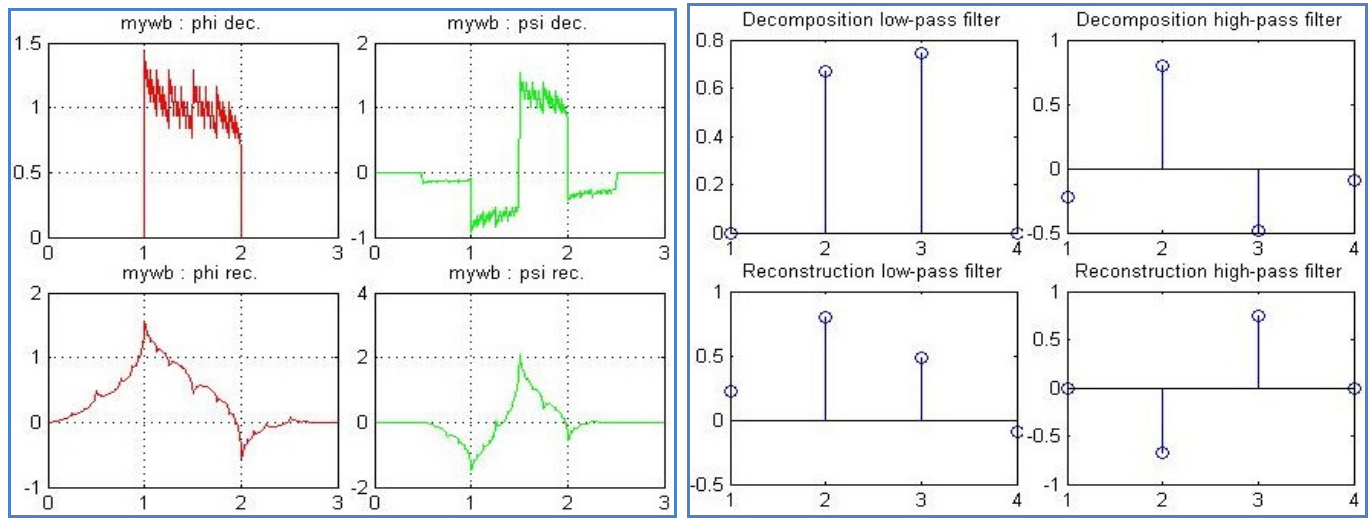

Figure 5. New Wavelets: phi, psi functions and four filters associated with NBior4

Table 1. PSNR values obtained with new wavelets

\begin{tabular}{|l|c|c|c|c|c|c|}
\hline \multicolumn{7}{|c|}{ PSNR (dB) } \\
\hline & Lena.jpg & Rice.jpg & ct.jpg & Pepper.jpg & moon.tif & office_1.jpg \\
\hline NBior1 & 31.64 & 32.81 & 33.30 & 30.95 & 34.27 & 37.56 \\
\hline NBior2 & 29.90 & 29.37 & 30.54 & 28.75 & 31.79 & 36.61 \\
\hline NBior3 & 26.70 & 27.01 & 26.03 & 24.95 & 27.62 & 34.17 \\
\hline NBior4 & 30.00 & 29.94 & 31.67 & 28.32 & 33.32 & 37.36 \\
\hline
\end{tabular}

Table 2. PSNR values obtained with new wavelets (contd. From Table 1)

\begin{tabular}{|l|c|c|c|c|c|c|}
\hline \multicolumn{7}{|c|}{ PSNR (dB) } \\
\hline & office_2.jpg & gantrycrane.png & circuit.tif & glass.tif & kids.tif & mandi.tif \\
\hline NBior1 & 33.51 & 28.85 & 32.93 & 32.89 & 40.64 & 32.88 \\
\hline NBior2 & 32.17 & 27.45 & 30.91 & 31.73 & 39.35 & 29.75 \\
\hline NBior3 & 29.24 & 24.63 & 27.32 & 30.95 & 36.61 & 27.47 \\
\hline NBior4 & 33.18 & 29.83 & 31.07 & 30.43 & 39.78 & 31.47 \\
\hline
\end{tabular}


Signal \& Image Processing : An International Journal (SIPIJ) Vol.4, No.6, December 2013

Table 3. CR values obtained with new wavelets

\begin{tabular}{|l|c|c|c|c|c|c|}
\hline \multicolumn{7}{|c|}{ CR (bpp) } \\
\hline & Lena.jpg & Rice.jpg & ct.jpg & Pepper.jpg & moon.tif & office_1.jpg \\
\hline NBior1 & 2.97 & 3.12 & 3.53 & 3.07 & 3.09 & 3.63 \\
\hline NBior2 & 2.98 & 2.88 & 3.53 & 3.08 & 3.10 & 3.64 \\
\hline NBior3 & 2.67 & 2.79 & 3.47 & 2.71 & 2.85 & 3.36 \\
\hline NBior4 & 2.62 & 2.75 & 3.44 & 2.69 & 2.82 & 3.36 \\
\hline
\end{tabular}

Table 4. CR values obtained with new wavelets (contd. From Table 3)

\begin{tabular}{|l|c|c|c|c|c|c|}
\hline \multicolumn{7}{|c|}{ CR (bpp) } \\
\hline & office_2.jpg & gantrycrane.png & circuit.tif & glass.tif & kids.tif & mandi.tif \\
\hline NBior1 & 3.00 & 3.01 & 2.94 & 3.46 & 3.93 & 3.09 \\
\hline NBior2 & 3.00 & 3.01 & 2.95 & 3.47 & 3.95 & 2.85 \\
\hline NBior3 & 2.75 & 2.82 & 2.55 & 3.11 & 3.63 & 2.72 \\
\hline NBior4 & 2.74 & 2.80 & 2.56 & 3.04 & 3.58 & 2.69 \\
\hline
\end{tabular}

The new wavelet NBior1 is producing a PSNR around 33dB, while the CR is around 3bpp. On some images it is producing a PSNR of even $40 \mathrm{~dB}, 37 \mathrm{~dB}$ and $34 \mathrm{~dB}$. With the new wavelet NBior2, the PSNR is around $31 \mathrm{~dB}$ and CR is around 3bpp. In the case of NBior3, the PSNR is around $28 \mathrm{~dB}$ and CR is around 3bpp. With NBior4, the PSNR is around 32dB and CR is around 3bpp. The average values of PSNR, CR and PC [9] are given in table 5.

Table 5. Average PSNR, CR and PC values

\begin{tabular}{|c|c|c|c|}
\hline & PSNR & CR & PC \\
\hline NBior1 & 33.52 & 3.24 & 108.67 \\
\hline NBior2 & 31.53 & 3.20 & 101.14 \\
\hline NBior3 & 28.56 & 2.95 & 84.45 \\
\hline NBior4 & 32.20 & 2.92 & 94.33 \\
\hline
\end{tabular}

The proposed wavelets are compared with traditional wavelets. The PSNR, CR and PC values obtained using traditional and proposed wavelets are plotted in the figures 6, 7 and 8 .

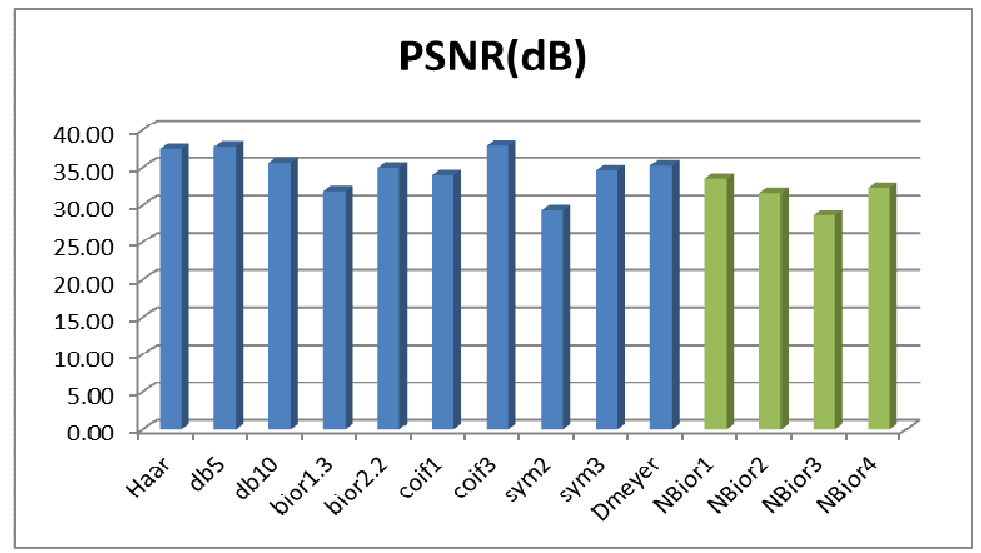

Figure 6. PSNR values of traditional and proposed wavelets 
Signal \& Image Processing : An International Journal (SIPIJ) Vol.4, No.6, December 2013

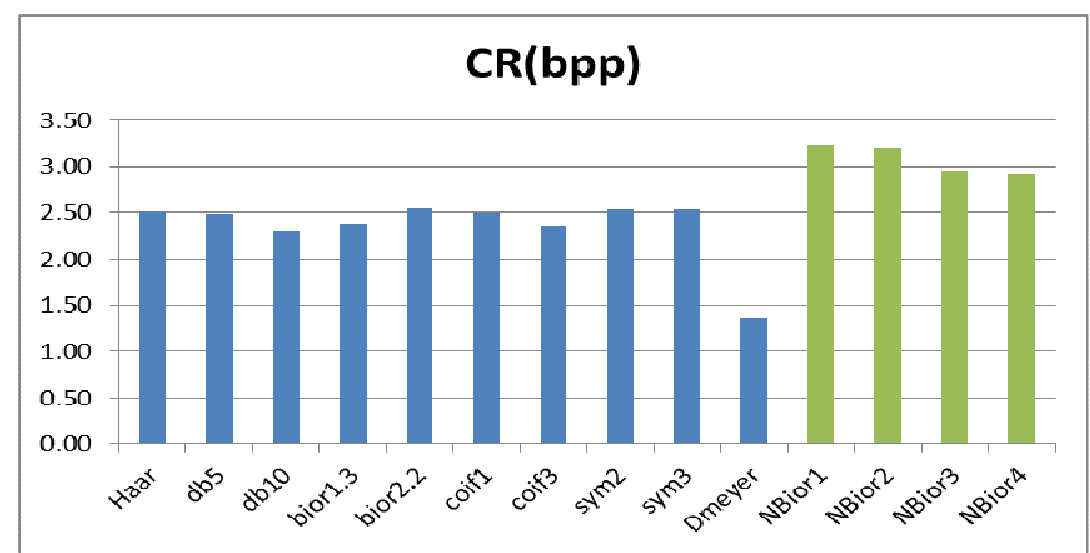

Figure 7. CR values of traditional and proposed wavelets

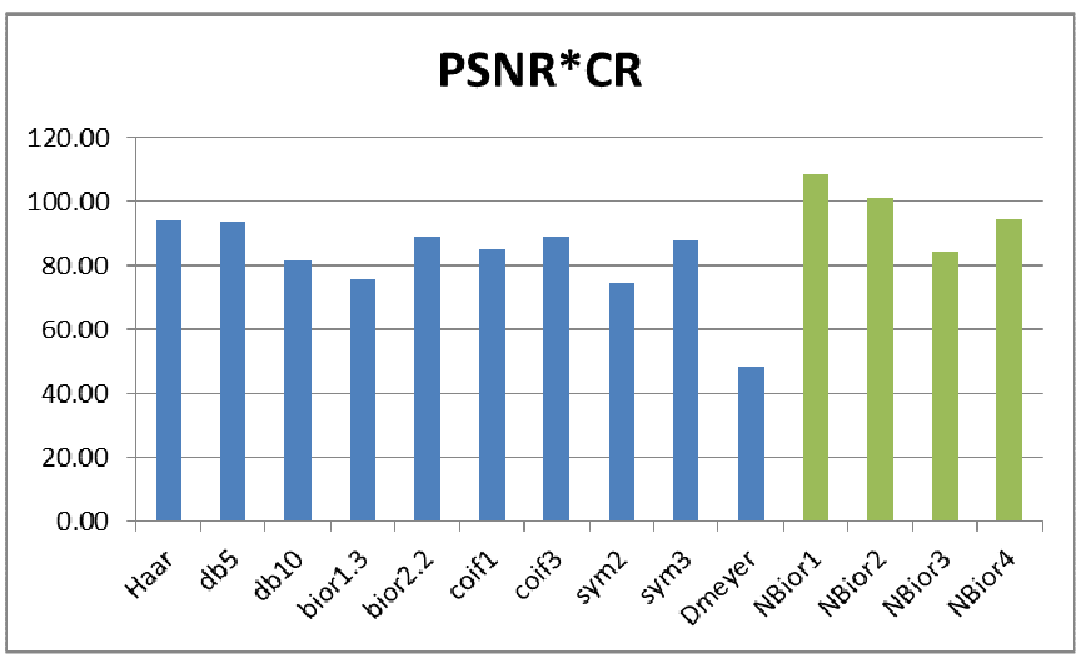

Figure 8 . PSNR*CR values of traditional and proposed wavelets

From the above figures, it is clear that the PSNR is almost in the same range with both traditional and proposed wavelets and CR is clearly high with proposed wavelets compared with that of traditional wavelets. The PSNR ${ }^{*} \mathrm{CR}$ value of Nbior1 is around 108 which is greater than that of all other proposed and traditional wavelets. The performance of new wavelets on binary images is presented in tables 6 and 7.

Table 6. PSNR values obtained with new wavelets on binary images

\begin{tabular}{|l|c|c|c|c|}
\hline \multicolumn{5}{|c|}{ PSNR (dB) } \\
\hline & blobs.png & circle.png & circbw.tif & logo.tif \\
\hline NBior1 & 62.45 & 69.69 & 61.54 & 64.33 \\
\hline NBior2 & 60.21 & 66.21 & 56.85 & 56.96 \\
\hline NBior3 & 61.00 & 63.00 & 55.44 & 58.21 \\
\hline NBior4 & 61.73 & 67.95 & 59.91 & 62.65 \\
\hline
\end{tabular}


Signal \& Image Processing : An International Journal (SIPIJ) Vol.4, No.6, December 2013

Table 7. CR values obtained with new wavelets on binary images

\begin{tabular}{|l|c|c|c|c|}
\hline \multicolumn{5}{|c|}{ CR (bpp) } \\
\hline & blobs.png & circle.png & circbw.tif & logo.tif \\
\hline NBior1 & 17.27 & 17.00 & 15.73 & 15.80 \\
\hline NBior2 & 17.27 & 17.00 & 15.73 & 15.80 \\
\hline NBior3 & 17.00 & 16.99 & 15.51 & 15.74 \\
\hline NBior4 & 17.14 & 17.00 & 15.67 & 15.80 \\
\hline
\end{tabular}

The PSNR values with proposed wavelets on binary images are around $60 \mathrm{~dB}$ and $\mathrm{CR}$ around 16bpp.

\section{Conclusions}

In this paper, four new biorthogonal wavelets are proposed. The proposed wavelets are used in representing image in a suitable format to apply SPIHT coding.The simulation results proved that the compression performance of proposed wavelets is superior to that of traditional wavelets. The PSNR obtained with traditional wavelets is around $35 \mathrm{~dB}$ and CR is around $2.5 \mathrm{bpp}$. While, with the proposed wavelets PSNR is around 32dB and CR is around 3bpp. The PSNR with proposed wavelets is slightly lesser than that of traditional wavelets, but compression ratio is higher enough so that the PSNR*CR is 9 more than that of traditional wavelets. It is expected that if the lifting version of proposed wavelets gives even better results and those results will comparable to that of the lifting version of traditional wavelets.

\section{REFERENCES}

[1] M. Antonini, M. Barlaud, P. Mathieu, and I. Daubechies., "Image coding using wavelet transform”, IEEE Trans. Image Proc., 1(2):205-220, April 1992.

[2] H. B"olcskei and F. Hlawatsch., "Gabor Analysis and Algorithms: Theory and Applications", chapter Oversampled modulated filter banks, pages 295-322. Birkh"auser, Boston, MA, 1998.

[3] J. Kova cevi'c and M. Vetterli., "Nonseparable multidimensional perfect reconstruction filter banks and wavelet bases for $\mathrm{R}^{\mathrm{n}}$, IEEE Trans. Inform. Sp. iss. on Wavelet Transforms and Multiresolution Signal Analysis, 38(2):533-555, March 1992.

[4] S. Mallat., "A Wavelet Tour of Signal Processing”, Academic Press, second edition, 1999.

[5] A. Sandryhaila, A. Chebira, C. Milo, J. Kovǎcevi'c, and M. P"uschel., "Systematic construction of real lapped tight frame transforms", IEEE Trans. Signal Proc., 58(5):2256-2567, May 2010.

[6] G. Strang and T. Nguyen., "Wavelets and Filter Banks", Wellesley Cambridge Press, Boston, MA, 1996.

[7] H. Yoo, and J. Jeong, "Signal-dependent wavelet transform and application to lossless image compression”, Electronics Letters, Vol. 38, no 4, pags. 170-172, February 2002.

[8] M. Unser, and T. Blu, "Mathematical properties of the JPEG2000 wavelet filters", IEEE Transactions on Image Processing, Vol. 12, no 9, pags. 1080-1090, September 2003.

[9] M. Santhosh, B. Stephen Charles, Jr., M. N. Giri Prasad, "Image Representation by First Generation Wavelets and Its Application to Compression", CiiT Journal of Digital Image Processing, vol. , Month, Year. 
Signal \& Image Processing : An International Journal (SIPIJ) Vol.4, No.6, December 2013

AUTHORS

M. Santhosh, born on $1^{\text {st }}$ July 1975 , received B.Tech degree from Sri Venkateswara University, Tirupati, India in 1997, M.Tech degree from Maharaj Sayaji Rao University, Baroda in 1999. She has started her career as an Asst. Prof., in AITS, Rajampet in 1999 and presently working as an Asso. Prof., in Stanley Stephen College of Engineering and Technology, Kurnool.She has more than 13 years of reacing experience. Her research interests include image representation techniques, compression and wavelets.

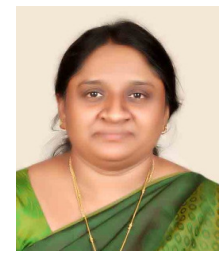

Dr. B. Stephen Charles (India) born on the 9th of August 1965. He received Ph.d degree in Electronics \& Communication Engineering from Jawaharlal Nehru Technological University, Hyderabad in 2001. His area of interest is Digital signal Processing. He received his B.Tech degree in Electronics and Communication Engineering from Nagarjuna University, India in 1986. He started his carrier as Assistant professor in Karunya institute of technology during 1989 to 1993, later joined as Associate Professor in K. S. R. M. College of Engg. During 1993 to 2001 after that he worked as Principal of

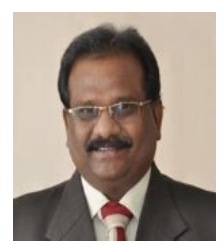
St. John's College of Engineering \& Technology during 2001 to 2007 and now he is the Secretary, Correspondent and Principal in Stanley Stephen College of Engineering \& Technology, Kurnool. He has more than 25 years of teaching and research experience. He published more than 40 research papers in national and international journals and more than 30 research papers in national and international conferences. He is a member of Institute of Engineers and ISTE.

Dr. M.N. Giri Prasad received his B.Tech degree from J.N.T University College of Engineering, Anantapur, Andhrapradesh, India in 1982. M.Tech degree from Sri Venkateshwara University, Tirupati, Andhra Pradesh, India in 1994 and Ph.D degree from J.N.T. University, Hyderabad, Andhra Pradesh, Indian in 2003. Presently he is working as a Professor in the Department of Electronics and Communication at J.N.T University College of Engineering Anantapur, Andhrapradesh, India. He has more than 25 years of teaching and research experience. He has published more than 50 papers in

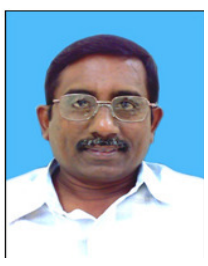
national and international journals and more than 30 research papers in national and international conferences. His research areas are Wireless Communications and Biomedical instrumentation, digital signal processing, VHDL coding and evolutionary computing. He is a member of ISTE, IE \& NAFEN. 